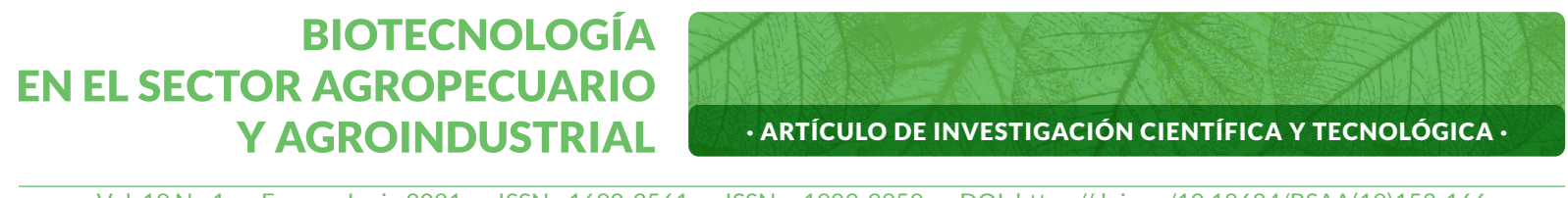

\title{
Evaluación de parámetros zootécnicos en terneros suplementados con un alimento fermentado en estado sólido*
}

\section{Evaluation of zootechnical parameters in calves supplemented with a fermented food in solid state}

\section{Avaliação de parâmetros zootécnicos em berços suplementados com alimento fermentado em estado sólido}

RODRÍGUEZ-SALGADO, ANGELA-MIREYA; RODRÍGUEZ-MOLANO, CARLOS-EDUARDO; BORRAS-SANDOVAL, LUIS-MIGUEL²

Historial del Artículo

Recibido para evaluación: 17 de Abril 2020.

Aprobado para publicación: 20 de Octubre 2020

* Título del proyecto de origen: "Evaluación del desarrollo ruminal y de algunos parámetros productivos en terneros alimentados con una dieta obtenida por fermentación en estado sólido (FES) papa: zanahoria". Financiación: Universidad Pedagógica y Tecnológica de Colombia y COLCIENCIAS. Culminación: diciembre de 2019.

1 Universidad Pedagógica y Tecnológica de Colombia, Facultad de Ciencias Agropecuarias, Grupo de investigación en Bioquímica y Nutrición Animal (GIBNA). MSc. en Ciencias Biológicas. Tunja, Colombia. https://orcid.org/0000-0002-0862-3478

2 Universidad Pedagógica y Tecnológica de Colombia, Facultad de Ciencias Agropecuarias, Grupo de investigación en Bioquímica y Nutrición Animal (GIBNA). Ph.D. en Ciencias Veterinarias. Tunja, Colombia. https://orcid.org/0000-0002-3284-027X

Correspondencia: carlos.rodriguez@uptc.edu.co

Cómo citar este artículo: RODRÍGUEZ-SALGADO, ANGELA-MIREYA; RODRÍGUEZMOLANO, CARLOS-EDUARDO, BORRAS-SANDOVAL, LUIS-MIGUEL .Evaluación de parámetros zootécnicos en terneros suplementados con un alimento fermentado en estado sólido. Biotecnología en el sector agropecuario y agroindustrial, v. 19, n. 1, 2021, p. 153-166.

Doi: https://doi.org/10.18684/BSAA(19)153-166 


\section{RESUMEN}

El crecimiento de la población mundial ha causado un aumento en la demanda de alimentos de origen animal, en donde el ganado bovino se ha convertido en una de las especies más importantes económicamente hablando, a raíz de los altos costos de concentrados se ha generado interés en la búsqueda de alternativas de alimentación. El objetivo de este trabajo fue determinar el comportamiento de algunos parámetros zootécnicos en terneros suplementados con diferentes inclusiones de FES papa- zanahoria. Se establecieron cuatro grupos con una alimentación base de leche y forraje, dos grupos fueron suplementados con 6 y $9 \mathrm{~g} / \mathrm{kg} / \mathrm{PV}$ de alimento FES papa- zanahoria, un grupo con 6 $g$ de concentrado balanceado y un grupo control. Las variables evaluadas fueron ganancia de peso, altura de la cruz y consumo de alimento. Los animales del T2 y T3, alcanzaron un peso final estadísticamente superior $(p<0,05)$ en relación a los animales del grupo control; la mayor altura a la cruz se logró con el T2, reportando una altura de $85 \mathrm{~cm}$. La suplementación con $6 \mathrm{~g}$ de alimento FES, resulta ser una alternativa de alimentación, al obtener mejores resultados en los parámetros zootécnicos y siendo más económica.

\section{ABSTRACT}

The growth of the world population has caused an increase in the demand for food of animal origin, where cattle have become one of the most important species economically speaking, due to the high costs of concentrates, interest has been generated in the search for feeding alternatives. The objective of this work was to determine the behavior of some zoo technical parameters in calves supplemented with different of FES potato-carrot inclusions. Four groups were established with a basic diet of milk and forage, two groups were supplemented with 6 and $9 \mathrm{~g} / \mathrm{kg} / \mathrm{PV}$ of FES-carrot feed, one group with $6 \mathrm{~g}$ of balanced concentrate and the control group. The variables evaluated were weight gain, height at the withers and food consumption. The animals of T2 and T3 reached a statistically higher final weight $(p<0,05)$ in relation to the animals of the control group; the highest height at the withers was achieved with the T2, reporting a height of $85 \mathrm{~cm}$. Supplementation with $6 \mathrm{~g}$ of FES feed turns out to be a feeding alternative, obtaining better results in zoo technical parameters and being more economical.

\section{RESUMO}

O crescimento da população mundial causou um aumento na demanda por alimentos de origem animal, onde o gado se tornou uma das espécies mais importantes economicamente falando, Devido ao alto custo dos concentrados, foi gerado interesse na busca de alternativas alimentares. O objetivo deste trabalho foi determinar o comportamento de alguns parâmetros zootécnicos em bezerros suplementados com diferentes inclusões de cenoura-batata FES. Quatro grupos foram estabelecidos com uma dieta básica de leite e forragem, dois grupos foram suplementados com 6 e $9 \mathrm{~g} / \mathrm{kg} / \mathrm{PV}$ de ração FES-cenoura, um grupo com 6 $g$ de concentrado balanceado e um grupo controle. As variáveis avaliadas foram ganho de peso, estatura na cernelha e consumo alimentar. Os animais de T2 $e$

\section{PALABRAS CLAVES:}

Alimentación animal; Forraje; Papa; Zanahoria; Alternativa nutricional; Alimento conservado.

\section{KEYWORDS:}

Animal feed; Forage; Potato; Carrot; Nutritional alternative; Preserved food.

\section{PALAVRAS-CHAVE:}

Alimentação animal; Forragem; Batata; Cenoura; Alternativa nutricional; Alimento conservado. 
T3 atingiram um peso final estatisticamente maior $(p<0,05)$ em relação aos animais do grupo controle; a altura mais alta na cernel ha foi alcançada com o T2, relatando uma altura de $85 \mathrm{~cm}$. A suplementação com $6 \mathrm{~g}$ de alimentação FES acaba sendo uma alternativa alimentar, obtendo melhores resultados em parâmetros zootécnicos e sendo mais econômica.

\section{INTRODUCCIÓN}

El crecimiento acelerado y continuo de la población mundial, la concentración en las ciudades y el incremento de los ingresos ha generado el aumento de la demanda de alimentos, presentándose implicaciones de alto impacto en la producción, ya que se deberá producir más alimentos de origen animal con baja disponibilidad de recursos naturales [1]. La producción de carne bovina en Colombia integra uno de los sectores de mayor importancia económica, que le garantiza al país el autoabastecimiento de productos cárnicos, sin embargo, la baja tasa de crecimiento de los animales, altos costos de concentrados y cambio climático limita esta actividad.

La creciente demanda de productos animales brinda a todos los involucrados en la producción de rumiantes implementar el uso estratégico de la nutrición para mejorar la salud y la producción, ya que se estima que esta es uno de los factores más importantes en el desarrollo productivo de los animales, además de contribuir en hasta el $70 \%$ del costo total de producción [2], debido a esto, se ha visto la obligación de buscar e implementar continuamente estrategias nutricionales alternativas para la suplementación de proteína y materia seca, de calidad y económicamente rentables, supliendo los requerimientos nutricionales y mejorando los parámetros zootécnicos [3].

La elaboración de alimentos mediante fermentación en estado sólido (FES) constituye una alternativa viable, debido a que permite el aprovechamiento de subproductos de residuos agroindustriales, lo que contribuye a mejorar la dieta de los rumiantes, aumentando los indicadores productivos y reduciendo la contaminación ambiental [4]. En Boyacá, al ser un departamento con vocación agrícola, se evidencian cantidades representativas de residuos pos cosecha que generan pérdidas económicas para los productores y pueden actuar como agentes potencialmente contaminantes; por esta razón, se ha visto la necesidad de implementar tecnologías para su aprovechamiento llegándose a estudiar la inclusión de diferentes residuos de papa, zanahoria, manzana, durazno y chontaduro en un preparado FES como alternativa ecoeficiente para la alimentación animal [5,6,7,8,9].

La ingesta de alimento sólido en terneros es un factor importante en su desarrollo ruminal permitiendo realizar la transición de un animal pre-rumiante a rumiante funcional, la utilización de residuos agroindustriales, productos y subproductos agrícolas locales ricos en proteínas y nutrientes de alto valor energético, puede constituir una alternativa para mejorar el rendimiento animal, además de obtener reducción en los costos de producción, sin embargo, en la actualidad se evidencia controversia respecto a la composición y calidad del alimento iniciador. Teniendo en cuenta estas consideraciones, el objetivo de este trabajo fue determinar el efecto de la suplementación de inclusiones de FES papa-zanahoria sobre algunos parámetros zootécnicos en terneros.

\section{MÉTODO}

\section{Localización el estudio}

La investigación fue realizada en la finca Santa Bárbara, ubicada en el Municipio de Gachantivá, Departamento de Boyacá perteneciente a la provincia de Alto Ricaurte, ubicada $73^{\circ} 33^{\prime} 00^{\prime \prime}$ de longitud y $5^{\circ} 44^{\prime} 50^{\prime \prime}$ de latitud, a una altura de $2450 \mathrm{msnm}$, temperatura promedio de $16^{\circ} \mathrm{C}$ y humedad relativa del $64 \%$.

\section{Manejo de los animales}

Se seleccionaron terneros de diferentes cruces de Holstein $(n=16)$ provenientes de ganaderías especializadas, con 3 días de edad, con un criterio de selección de homogeneidad de peso. Se inició el suministro de leche a ra- 
zón de $4 \mathrm{~L}$ en dos tomas (6:00 y 18:00 horas) a una temperatura de 37 a $40^{\circ} \mathrm{C}$ por medio de un biberón especial para este tipo de trabajo.

A la semana de vida se inició un proceso de acostumbramiento a la dieta, proceso que tuvo una duración de 7 días, donde se inició con el suministro de 30 g/día de alimento FES y concentrado comercial para los tratamientos T2 y T3 respectivamente, con un aumento diario gradual hasta completar los $6 \mathrm{~g} / \mathrm{kg} / \mathrm{PV}$, para el T4 se suministró inicialmente $50 \mathrm{~g} /$ día de alimento FES con un aumento gradual diario igual hasta completar los $9 \mathrm{~g} /$ $\mathrm{kg} / \mathrm{PV}$, esto con el fin de garantizar el consumo de alimento en los diferentes tratamientos. Los animales fueron mantenidos bajo las mismas condiciones medio ambientales con una temperatura promedio de $16^{\circ} \mathrm{C}$ y humedad relativa de $64 \%$, manejando un modelo de semiestabulación, con el fin de tener un mejor control sobre las unidades experimentales; se contaban con pequeños potreros con una base forrajera de pasto estrella (Cynodon nlemfluensis), adicionalmente, se les suministró heno de pangola (Digitaria eriantha) y cuba 22 (Cuba CT-115); el consumo de materia seca en base en forraje se estimó en un 2,8\% de su peso vivo, el suministro de agua fue ad libitum y a todos los animales se les brindaron los cuidados necesarios para mantener su integridad y bienestar.

\section{Tratamientos}

Los terneros $(n=16)$ fueron asignados aleatoriamente a 4 tratamientos con el factor de inclusión de alimento FES y concentrado balanceado comercial, la suplementación se inició en la segunda semana de vida y se llevó hasta la semana 10 con el fin de coincidir con la fase de transición de los rumiantes.

Tratamiento 1 (T1) Control (Leche-Forraje). Suministro de leche y forraje a voluntad.

Tratamiento 2 (T2) Leche/Forraje/FES(6g/kg/PV). Suministro de leche y forraje como base, más $6 \mathrm{~g} / \mathrm{kg}$ de peso vivo de FES papa-zanahoria.

Tratamiento 3 (T3). Leche/Forraje/Concentrado(6g/kg/PV): Suministro de leche y forraje como base, más $6 \mathrm{~g} /$ kg de peso vivo de alimento concentrado comercial.

Tratamiento 4 (T4) Leche/Forraje/FES(9g/kg/PV). Suministro de leche y forraje como base, más $9 \mathrm{~g} / \mathrm{kg}$ de peso vivo de FES papa- zanahoria.

Como alimento concentrado (cuadro 1) se empleó un alimento extruido comercial para etapa de preiniciación e iniciación formulado para la crianza de terneras. En cuanto al FES (cuadro 1), se usó uno FES papa- zanahoria elaborado a razón de $25 \%$ papa, $25 \%$ zanahoria, $16 \%$ salvado de trigo, $16 \%$ repila de trigo, $5 \%$ palmiste, $2 \%$ urea, 0,5\% sulfato de magnesio, 0,5\% carbonato de calcio, $5 \%$ inoculo BAL y $5 \%$ Melaza, este alimento se usó a las 48 horas de preparado, con base en investigaciones previas que demuestran su estabilidad y calidad nutricional [9].

Cuadro 1. Análisis garantizado del Alimento FES y del concentrado balanceado comercial en base a materia seca.

\begin{tabular}{|l|c|c|}
\hline \multicolumn{1}{|c|}{ Nutriente } & $\begin{array}{c}\text { FES 25:25 papa:zanahoria } \\
\text { (\%) }\end{array}$ & $\begin{array}{c}\text { Concentrado Comercial }{ }^{\circledR} \\
\text { (\%) }\end{array}$ \\
\hline Proteína Cruda & 27,80 & 18,0 \\
\hline Grasa & 6,20 & 2,5 \\
\hline Fibra Cruda & 9,00 & 12,0 \\
\hline Cenizas & 6,60 & 10,0 \\
\hline Humedad & 52,54 & 13,0 \\
\hline Materia seca & 47,46 & 87,0 \\
\hline
\end{tabular}




\section{Parámetros productivos a evaluar}

Como parámetros zootécnicos se midió la ganancia de peso total, ganancia de peso diario, consumo de alimento y altura a la cruz. Estas mediciones se iniciaron antes de realizar la suplementación y continuaron cada quince días hasta completar el tiempo del experimento que correspondió a la semana 10 de vida.

Ganancia de peso. Los animales fueron pesados en una báscula electrónica en las horas de la mañana en ayuno. Con estos datos se determinó la ganancia diaria de peso (g/día) y la ganancia de peso total (kg) en el tiempo de estudio.

Altura a la cruz (cm). La medición se hizo con el animal en posición tal que los miembros se encontraran aplomados con el eje central. La medida se tomó con un hipómetro en forma perpendicular desde el piso hasta la porción más sobresaliente de los cartílagos de las escapulas (cruz) por la cara posterior de los miembros anteriores, siguiendo la metodología descrita por Inchausti y Tagle [10], con el fin de determinar su crecimiento en centímetros.

\section{Diseño experimental}

Se aplicó un diseño completamente aleatorizado. Los datos fueron sometidos a los supuestos de normalidad a través del estadístico de Shapiro-Wilk y homogeneidad por prueba de Levene. Se utilizó la prueba de comparación de promedios de Tukey con un nivel de significancia del 5\%. El paquete estadístico a usado fue SPSS versión 23.

\section{RESULTADOS}

\section{Calidad nutricional de los alimentos}

Se evidenció que, en cuanto a los aportes nutricionales con base en la materia seca, el alimento obtenido por FES fue de mejor calidad que el concentrado balanceado comercial (Cuadro 1), mostrando mayor porcentaje de proteína, sin embargo, presentó una notable diferencia en la MS, motivo por el cual el consumo de alimento se calculó con base en la materia seca (CMS) y posteriormente se convirtió a consumo de alimento fresco (CAF) (Cuadro 2).

El alto contenido de proteína cruda en el alimento FES se relaciona con el aporte de nitrógeno dado por la úrea, a la reducción de carbohidratos y a la producción de proteínas microbianas durante el proceso de fermentación [11], sin embargo, estos niveles altos pueden relacionarse con la pérdida de materia seca permitiendo que la proteína pueda concentrarse [12], todo lo anterior evidenciado en este estudio. Este comportamiento fue comprobado por Fonseca et al. [5] quienes informaron que las diferentes inclusiones de zanahoria, nabo, tuza, morera y cascarilla de café mostraron un aumento promedio de cuatro veces el valor inicial a las 48 y 72 horas de fermentación; por otra parte, Caicedo et al. [13] indicaron que el contenido de proteína del tubérculo de taro presentó un incremento de $13,95 \%$ en relación con el tubérculo en estado natural que fue de $8,48 \%$, sin embargo, Fonseca et al. [14] reportaron que bajos niveles de proteína asociados al almidón acelera el proceso de gelatinización lo cual es ideal para aumentar la digestibilidad de estos nutrientes.

\section{Consumo de alimento}

El suministro del concentrado balanceado estuvo acorde a lo reportado por Yohe et al. [15] quienes señalaron

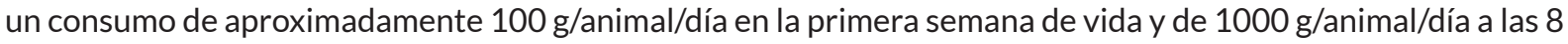
a 10 semanas, la cantidad de alimento suministrado se ajustó cada vez que se realizaba un pesaje.

En el cuadro 2 se puede observar que a medida que avanza la edad de los animales, en el T4 se evidencia un mayor consumo de alimento presentándose diferencias estadísticas significativas $(p<0,05)$ con respecto a los demás tratamientos. 
Cuadro 2. Consumo del FES papa: zanahoria y concentrado balanceado comercial.

\begin{tabular}{|c|c|c|c|c|}
\hline Intervalo (días) & Tratamiento & $\begin{array}{c}\text { Peso Inicial } \\
(\mathrm{Kg})\end{array}$ & $\mathrm{CMS}^{*}(\mathrm{~g})$ & $\mathrm{CAF}^{* *}(\mathrm{~g})$ \\
\hline \multirow{4}{*}{$\begin{array}{l}\text { Intervalo } 1 \\
(14-27 d)\end{array}$} & $\mathrm{T} 1^{* * *}$ & 34,83 & 0 & 0 \\
\hline & T2 & 35,75 & $214,5 \mathrm{a}$ & $451,6 b$ \\
\hline & T3 & 36,15 & $216,9 a$ & $249,3 a$ \\
\hline & T4 & 35,88 & $322,9 \mathrm{~b}$ & $679,8 \mathrm{c}$ \\
\hline \multirow{4}{*}{$\begin{array}{l}\text { Intervalo } 2 \\
\text { (28-41d) }\end{array}$} & $\mathrm{T} 1^{* * *}$ & 40,68 & 0 & 0 \\
\hline & T2 & 41,73 & $250,4 a$ & $527,1 \mathrm{~b}$ \\
\hline & T3 & 41,9 & $251,4 a$ & $289,0 \mathrm{a}$ \\
\hline & T4 & 42,63 & $383,7 \mathrm{~b}$ & $807,7 \mathrm{c}$ \\
\hline \multirow{4}{*}{$\begin{array}{l}\text { Intervalo } 3 \\
(42-55 d)\end{array}$} & $\mathrm{T} 1^{* * *}$ & 40,95 & 0 & 0 \\
\hline & $\mathrm{T} 2$ & 45,9 & $275,4 \mathrm{a}$ & $579,8 \mathrm{~b}$ \\
\hline & T3 & 46,18 & $277,1 \mathrm{a}$ & $318,5 a$ \\
\hline & T4 & 45,75 & $411,8 \mathrm{~b}$ & $866,8 \mathrm{c}$ \\
\hline \multirow{4}{*}{$\begin{array}{l}\text { Intervalo } 4 \\
(56-70 \mathrm{~d})\end{array}$} & $\mathrm{T} 1^{* * *}$ & 41,63 & 0 & 0 \\
\hline & T2 & 50,55 & $303,3 a$ & $638,5 b$ \\
\hline & T3 & 51,73 & $310,4 \mathrm{~b}$ & $356,8 \mathrm{a}$ \\
\hline & T4 & 50,43 & $453,9 c$ & $955,5 \mathrm{c}$ \\
\hline
\end{tabular}

${ }^{*}$ Consumo de materia seca

${ }^{* *}$ Consumo de alimento fresco

***No se calculó CMS y CAF debido a que no consumieron una dieta suplementaria Promedios con letras distintas en la columna, indican una diferencia estadística significativa según la prueba de Tukey $(P<0,05)$.

Para el primer intervalo es importante aclarar que los individuos ya habían recibido 7 días de acostumbramiento a la dieta, donde todos los animales consumieron la cantidad correspondiente para cada tratamiento sin presentar rechazo a los mismos; sin embargo, los animales del T4 no consumieron la totalidad del alimento ofrecido, a diferencia de los animales de los otros tratamientos en donde el consumo fue total; este mismo comportamiento se evidenció para el intervalo 2 y 3 ; finalizando la etapa experimental es decir en el intervalo 4, los animales del T4 consumieron todo el alimento.

En este estudio se usaron dosis bajas del alimento FES debido a que la presentación de un pH bajo en rumiantes en etapa de transición puede llegar a ser perjudicial para su desempeño, aumentando el riesgo de acidosis ruminal y la disminución de la ingesta de alimentación inicial [16], además de generar una mayor demanda de energía que reduciría la cantidad de energía disponible para el crecimiento, afectando la eficiencia de la alimentación [17], lo que se evidenció con la dosis más alta del alimento FES.

Los datos de consumo con base en MS fueron inferiores a los reportados por otros investigadores que señalan consumos de $340 \mathrm{~g} /$ día aproximadamente en las primeras semanas de vida del animal y $1720 \mathrm{~g} /$ día post-destete [18]; por otro lado, Castro \& Elizondo [19] reportan consumos a la semana 8 de $7030 \mathrm{~g} / \mathrm{semana}$ de alimento en formato harina (1004,2 g/día), $5856 \mathrm{~g} / \mathrm{semana}$ de concentrado en formato pellet (836,57 g/día) y de $6086 \mathrm{~g} /$ semana en concentrados balanceados en formato extrusado, correspondientes a 869,4 g/día.

Adicionalmente, se conoce que el consumo de alimento está estrechamente ligado con la presentación y composición del mismo tanto del tamaño como la frecuencia de alimentación; se ha informado que partículas finas genera una menor ingesta en relación con partículas de mayor tamaño en terneros [20], además la suplementación con forraje es un factor importante en la mejora del consumo [21]; por otro lado, Mirzaei et al. [22] demostraron que la suplementación con alimentos fermentados como el ensilaje de maíz mostraron una mayor ingesta inicial en relación con el heno de alfalfa e iniciadores granulados. Sin embargo, en otros estudios se ha demostrado que no se evidencia interacción entre la forma física de las dietas iniciales y la ingesta inicial del alimento [23]. 


\section{Peso de los animales}

El promedio de los pesos iniciales no mostró diferencias estadísticamente significativas $(p<0,05)$, sin embargo, a las 10 semanas de vida los animales de T2 y T3 alcanzaron un peso final estadísticamente superior $(p<0,05)$ en relación con el grupo control; en tanto que T4 no mostró diferencias con los otros tratamientos (Cuadro 3).

Cuadro 3. Pesaje de los animales.

\begin{tabular}{|l|l|l|l|l|l|}
\hline \multicolumn{1}{|c|}{ Tratamiento } & \multicolumn{1}{|c|}{ Peso Inicial } & \multicolumn{1}{|c|}{ Peso 28 días } & \multicolumn{1}{c|}{ Peso 42 días } & \multicolumn{1}{c|}{ Peso 56 días } & \multicolumn{1}{c|}{ Peso Final } \\
\hline T1 & $34,83 \pm 1,83 \mathrm{a}$ & $40,68 \pm 0,58 \mathrm{a}$ & $40,95 \pm 1,08 \mathrm{a}$ & $41,63 \pm 0,53 \mathrm{a}$ & $45,78 \pm 2,07 \mathrm{~b}$ \\
\hline T2 & $35,75 \pm 2,05 \mathrm{a}$ & $41,73 \pm 1,96 \mathrm{a}$ & $45,90 \pm 1,93 \mathrm{a}$ & $50,55 \pm 2,17 \mathrm{a}$ & $59,00 \pm 2,47 \mathrm{a}$ \\
\hline T3 & $36,15 \pm 2,63 \mathrm{a}$ & $41,90 \pm 3,48 \mathrm{a}$ & $46,18 \pm 3,99 \mathrm{a}$ & $51,73 \pm 4,39 \mathrm{a}$ & $58,30 \pm 4,82 \mathrm{a}$ \\
\hline T4 & $35,880,80 \mathrm{a}$ & $42,63 \pm 0,93 \mathrm{a}$ & $45,75 \pm 0,58 \mathrm{a}$ & $50,43 \pm 0,61 \mathrm{a}$ & $56,9 \pm 1,14 \mathrm{ab}$ \\
\hline
\end{tabular}

Promedios con letras distintas en la columna, indican una diferencia estadística significativa según la prueba de Tukey $(P<0,05) ; \pm$ error estándar $(n=4)$

Beiranvand et al. [18] reportaron pesos que oscilaron entre 61,37 y $65,58 \mathrm{~kg}$ al destete con diferentes Interacciones de heno de alfalfa y propionato de sodio; por otro lado, con una alimentación de leche mediante un procedimiento convencional y descendente, se obtuvieron pesos de 65 y 79,11 kg a la séptima semana de vida respectivamente [24]; 66,61 y 73,24 kg con la adición de agua en el concentrado [25] y 60,4 y 59,4 kg con avena molida y entera a la 5 semana de vida [26], datos que fueron superiores a los obtenidos en este estudio. Sin embargo, Castro y Elizondo [19] reportaron pesos que coincidieron, que oscilaron entre 49 y $58 \mathrm{~kg}$ a la 8 semana.

En contraste, Harris, et al. [27] mostraron un aumento significativo de los pesos de terneros suplementados con productos de fermentación de Saccharomycescerevisia obteniendo 60,1 kg a los 35 días de vida. Se ha conocido que alimentos fermentados ejercen un efecto positivo sobre los índices productivos de terneros como la ganancia de peso y la eficiencia alimenticia, permitiendo la acumulación de músculo corporal al proporcionar una mayor deposición de proteínas, todo esto debido a que el proceso de la fermentación mejora el valor nutricional al reducir agentes anti nutricionales, mejorar la digestibilidad y aumentar el contenido de proteínas y péptidos pequeños [28].

Las diferencias en los resultados pueden ser atribuidas a la variación en la composición de los alimentos suministrados, como relación forraje-concentrado, frecuencia de alimentación, tamaño de la partícula, además de las condiciones ambientales, edad y raza del animal. No obstante, es conocido que la cantidad y calidad del forraje suministrado es un factor importante en el desarrollo de los terneros ya que este puede mejorar el consumo de alimento sólido, la fermentación ruminal y el aumento del peso [29].

\section{Ganancia diaria de peso}

En el primer pesaje no se evidenciaron diferencias $(p<0,05)$ en las ganancias, sin embargo, en los pesajes a los 42 y 56 días los T2, T3 y T4, mostraron un aumento $(p<0,05)$ con respecto al grupo control. Para el pesaje a los 70 días, el T2 logró los mejores resultados con una ganancia diaria de peso de 603,6 g/día ( $p<0,05)$ seguido del T3 con 469,6 g y T4 con 462,5 g/día. Para la ganancia diaria promedio, se observa que en los animales suplementados lograron valores superiores a los $450 \mathrm{~g}$ /día; a la vez, se evidencia que subir la dosis de FES no se traduce en un mayor rendimiento productivo, muy posiblemente se deba a alteraciones bioquímica en la cámara retículo ruminal (Cuadro 4).

En el Cuadro 4 se observa una notable disminución en la ganancia diaria de peso en el pesaje a los 42 días, esto pudo ser debido a la presencia de neumonías neonatales en los terneros, sin embargo, se observa que a los 70 días, los animales suplementados recuperaron los niveles de ganancia de peso. 
Cuadro 4. Ganancia diaria de peso.

\begin{tabular}{|c|c|c|c|c|c|}
\hline Tratamiento & Pesaje 28 días (g/d) & Pesaje 42 días (g/d) & Pesaje 56 días (g/d) & Pesaje 70 días (g/d) & Promedio (g/d) \\
\hline T1 & $365,6 \pm 78,1 \mathrm{a}$ & $17,2 \pm 32,6 \mathrm{~b}$ & $51,9 \pm 112,1 \mathrm{~b}$ & $296,4 \pm 147,7 \mathrm{~b}$ & $223,5 \pm 74,2 \mathrm{~b}^{*}$ \\
\hline T2 & $373,4 \pm 42,8 \mathrm{a}$ & $260,9 \pm 3,9 \mathrm{a}$ & $357,7 \pm 23,8 \mathrm{a}$ & $603,6 \pm 42,50 \mathrm{a}$ & $474,5 \pm 26,8 \mathrm{a}^{*}$ \\
\hline T3 & $359,4 \pm 52,9 \mathrm{a}$ & $267,2 \pm 62,3 \mathrm{a}$ & $426,9 \pm 31,4 \mathrm{a}$ & $469,6 \pm 32,2 \mathrm{ab}$ & $452 \pm 47,7 \mathrm{a}^{*}$ \\
\hline T4 & $419,5 \pm 10,8 \mathrm{a}$ & $195,3 \pm 25,6 \mathrm{a}$ & $359,6 \pm 13,0 \mathrm{a}$ & $462,5 \pm 71,5 \mathrm{ab}$ & $429,1 \pm 9,8 \mathrm{a}^{*}$ \\
\hline
\end{tabular}

Promedios con letras distintas en la columna, indican una diferencia estadística significativa según la prueba de Tukey $(P<0,05) ; \pm$ error estándar $(n=4)$.

* Diferencia estadística significativa según la prueba de Duncan $(P<0,05)$.

Silva et al. [30] indicaron que en la suplementación con leche y concentrado a voluntad la ganancia diaria fue mayor al aumentar los niveles de suministro de leche concentrado obteniendo 794 g/día y 886 g/día con las concentraciones superiores, lo que no concuerda con lo obtenido en este estudio ya que al aumentar la cantidad de suministro del alimento FES se observaron ganancias de peso inferiores; estos resultados pueden relacionarse con la posible presencia de diferencias en el $\mathrm{pH}$ ruminal, debido a que este alimento posee alta cantidad de carbohidratos fermentables lo que reduce rápidamente el $\mathrm{pH}$ ruminal y por ende la ingesta de alimentación conllevando a perdidas en la ganancia de peso [26].

En estudios previos con alimentos fermentados demostraron que la ganancia diaria de peso fue superior a la obtenida en este estudio, con ganancias de 740 g/día y 796,9 g/día en la 8 semana de vida [28,31]. Kim et al. [32] reportaron que terneros alimentados con una dieta a base de harina de soja fermentada mejoraron la ingesta de alimento, el rendimiento del crecimiento, con ganancias aproximadas de $960 \mathrm{~g} / \mathrm{día}$ a la semana 6 valor superior a lo observado en este estudio, esto debido a que los alimentos fermentados aportan más nutrientes. En este orden de ideas, se ha demostrado que animales alimentados con productos fermentados presentan mejores condiciones de salud [33,34], lo que explicaría las mayores ganancias de peso en los tratamientos T2, T3 y T4 en relación con el grupo control en el pesaje a los 42 y 56 días.

\section{Ganancia Final de peso}

Se observó que la ganancia de peso final durante el tiempo de estudio muestra diferencias estadísticamente significativas $(p<0,05)$ entre los grupos de animales que recibieron suplementación y el grupo control. Es de resaltar que la mayor ganancia de peso se obtuvo con el T2, con un valor de 23,25 kg (Cuadro 5.).

Cuadro 5. Ganancia de peso final.

\begin{tabular}{|c|c|}
\hline Tratamiento & Ganancia de peso final (Kg) \\
\hline T1 & $10,95 \pm 3,63 \mathrm{~b}$ \\
\hline T2 & $23,25 \pm 1,31 \mathrm{a}$ \\
\hline T3 & $22,15 \pm 2,33 \mathrm{a}$ \\
\hline T4 & $21,03 \pm 0,48 \mathrm{a}$ \\
\hline
\end{tabular}

Promedios con letras distintas en la columna, indican una diferencia estadística significativa según la prueba de Tukey $(P<0,05)$; \pm error estándar $(n=4)$. 


\section{Altura a la cruz}

El comportamiento de la altura a la cruz en la Figura 1 muestra un patrón de crecimiento para los cuatro tratamientos.

Figura 1. Altura promedio a la cruz.

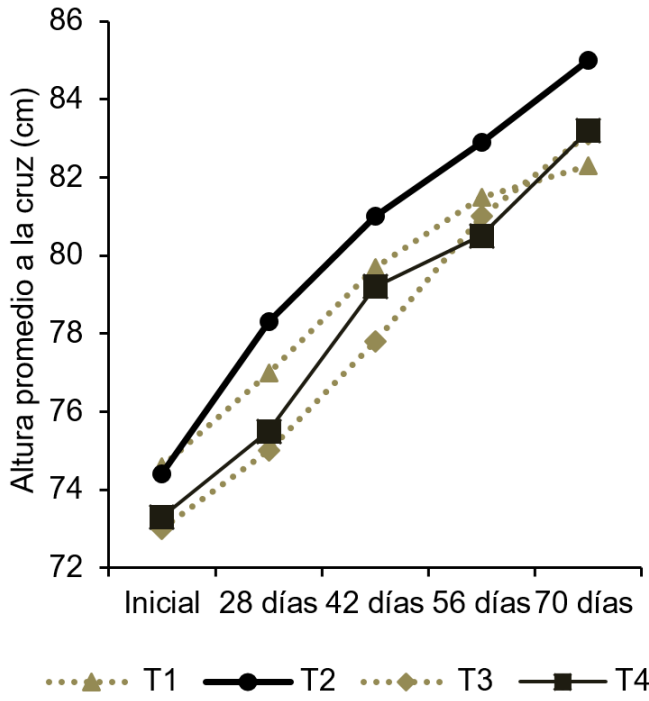

Los tratamientos suplementados presentaron mayores ganancias de altura $(p<0,05)$ con relación al grupo control, siendo el T2 y T3 los de mayor ganancia (Cuadro 6).

Cuadro 6. Ganancia promedio de la altura a la cruz.

\begin{tabular}{|c|c|c|c|c|c|}
\hline Tratamiento & $\begin{array}{c}\text { Ganancia Altura } \\
\text { 28 días }(\mathbf{c m})\end{array}$ & $\begin{array}{c}\text { Ganancia Altura } \\
\text { 42 días }(\mathbf{c m})\end{array}$ & $\begin{array}{c}\text { Ganancia Altura } \\
\text { 56 días }(\mathbf{c m})\end{array}$ & $\begin{array}{c}\text { Ganancia Altura } \\
\text { 70 días }(\mathbf{c m})\end{array}$ & $\begin{array}{c}\text { Ganancia Altura } \\
\text { Final }(\mathbf{C m})\end{array}$ \\
\hline T1 & 2,4 b & 2,2 b & $1,9 \mathrm{~b}$ & $0,8 \mathrm{~b}$ & 7,7 b \\
\hline T2 & 3,9 a & $2,8 \mathrm{a}$ & $1,9 \mathrm{~b}$ & $2,1 \mathrm{ab}$ & $10,6 \mathrm{a}$ \\
\hline T3 & 2,0 b & $2,8 \mathrm{a}$ & $3,3 \mathrm{a}$ & $2,1 \mathrm{ab}$ & $10,1 \mathrm{a}$ \\
\hline T4 & 2,2 b & $2,7 \mathrm{a}$ & $2,3 \mathrm{ab}$ & $2,7 \mathrm{a}$ & $9,9 \mathrm{a}$ \\
\hline
\end{tabular}

Promedios con letras distintas en la columna, indican una diferencia estadística significativa según la prueba de Tukey $(P<0,05) ; \pm$ error estándar $(n=4)$.

No obstante, estos valores difieren de los obtenidos por Khan et al. [35] quienes con consumo libre de heno alcanzaron 91,6 cm en la semana 8 y $95 \mathrm{~cm}$ en la semana 10; por otra parte, con la suplementación de harina de maíz fermentado a la semana 8 se obtuvo una altura promedio de $90,27 \mathrm{~cm}$ [31]. A diferencia, con la suplementación de $9 \%$ de harina de soja fermentada que obtuvo una altura de $81 \mathrm{~cm}$ en la semana 4 y $85 \mathrm{~cm}$ en la 8 semana, datos que concuerdan con los obtenidos por el T2; sin embargo, con 13,5\% de harina de soja se obtuvieron alturas de $83,42 \mathrm{~cm}$ y $87,15 \mathrm{~cm}[28]$.

\section{Consumo de alimento en relación al costo de la dieta}

En el cálculo del valor de la dieta suministrada a los animales no se tuvo en cuenta el costo de forraje y leche en ninguno de los tratamientos, debido a que este se realizó en base con el consumo de alimento concentrado y 
FES. El alimento FES papa:zanahoria tuvo un precio de $\$ 439 \mathrm{COP} / \mathrm{kg}$ de alimento, en comparación con el concentrado balanceado comercial con un precio de $\$ 1300 \mathrm{COP} / \mathrm{kg}$ de alimento; hasta este punto es notable que el alimento por FES tiene un costo $66,2 \%$ más bajo que el concentrado comercial; sumando a esto que su valor nutricional es más alto (Cuadro 7).

Los costos de alimentación relacionados en el Cuadro 8 corresponden a la sumatoria del costo de la alimentación durante el tiempo del experimento, los cuales se encuentran disgregados por intervalos de experimentación en el Cuadro 7.

Se evidencia que la alimentación con el T2, resulta ser más económica y productiva, ya que con esta se logran mejores ganancias de peso y de altura a la cruz; su uso representa un ahorro de $\$ 9081,2$ pesos en relación a la alimentación del T3.

La búsqueda de alimentos alternativos que suplan las necesidades nutricionales de los animales además de obtener resultados favorables en los parámetros productivos, es un factor importante en las producciones, ya que desde el punto de vista económico es imposible utilizar altos volúmenes de concentrados en la zona tropical.

Cuadro 7. Consumo de alimento en relación al costo de la dieta.

\begin{tabular}{|c|c|c|c|c|c|c|}
\hline Intervalo (días) & Tratamiento & $\begin{array}{l}\text { Peso Inicial } \\
(\mathrm{Kg})\end{array}$ & $\mathrm{CMS}^{*}(\mathrm{~g})$ & $\mathrm{CAF}^{* *}(\mathrm{~g})$ & $\begin{array}{c}\text { Costo \$ (COP) } \\
\text { Alimentación/ día }\end{array}$ & $\begin{array}{c}\text { Costo } \$(\mathrm{COP}) / \\
\text { intervalo de tiempo }\end{array}$ \\
\hline \multirow{4}{*}{$\begin{array}{l}\text { Intervalo } 1 \\
(14-27 d)\end{array}$} & $\mathrm{T} 1^{* * *}$ & 34,83 & 0 & 0 & 0 & 0 \\
\hline & $\mathrm{T} 2$ & 35,75 & $214,5 \mathrm{a}$ & $451,6 \mathrm{~b}$ & 197,7 & 3163,2 \\
\hline & T3 & 36,15 & $216,9 a$ & $249,3 \mathrm{a}$ & 324,1 & 5185,7 \\
\hline & T4 & 35,88 & $322,9 b$ & $679,8 \mathrm{c}$ & 297,6 & 4762,1 \\
\hline \multirow{4}{*}{$\begin{array}{l}\text { Intervalo } 2 \\
(28-41 d)\end{array}$} & T1 ${ }^{* * *}$ & 40,68 & 0 & 0 & 0 & 0 \\
\hline & $\mathrm{T} 2$ & 41,73 & $250,4 a$ & $527,1 \mathrm{~b}$ & 230,8 & 3230,8 \\
\hline & T3 & 41,9 & $251,4 a$ & $289,0 \mathrm{a}$ & 375,7 & 5259,2 \\
\hline & T4 & 42,63 & $383,7 b$ & $807,7 \mathrm{c}$ & 353,6 & 4950,7 \\
\hline \multirow{4}{*}{$\begin{array}{l}\text { Intervalo } 3 \\
\text { (42-56 d) }\end{array}$} & T1 ${ }^{* * *}$ & 40,95 & 0 & 0 & 0 & 0 \\
\hline & $\mathrm{T} 2$ & 45,9 & $275,4 a$ & $579,8 \mathrm{~b}$ & 253,8 & 3299,8 \\
\hline & T3 & 46,18 & $277,1 \mathrm{a}$ & $318,5 \mathrm{a}$ & 414,0 & 5382,4 \\
\hline & $\mathrm{T} 4$ & 45,75 & $411,8 \mathrm{~b}$ & $866,8 \mathrm{c}$ & 379,5 & 4933,5 \\
\hline \multirow{4}{*}{$\begin{array}{l}\text { Intervalo } 4 \\
\text { (56-70 d) }\end{array}$} & $\mathrm{T} 1^{* * *}$ & 41,63 & 0 & 0 & 0 & 0 \\
\hline & $\mathrm{T} 2$ & 50,55 & 303,3 a & $638,5 \mathrm{~b}$ & 279,5 & 4472,7 \\
\hline & T3 & 51,73 & 310,4 b & $356,8 \mathrm{c}$ & 463,8 & 7420,6 \\
\hline & $\mathrm{T} 4$ & 50,43 & $453,9 c$ & $955,5 \mathrm{a}$ & 418,3 & 6693,2 \\
\hline
\end{tabular}

${ }^{*}$ Consumo de materia seca

${ }^{* *}$ Consumo de alimento fresco

*** No se calcularon CMS, CAF y costos debido a que no consumieron una dieta suplementaria

Promedios con letras distintas en la columna, indican una diferencia estadística significativa según la prueba de Tukey $(P<0,05)$.

Cuadro 8. Costo de la alimentación en el tiempo de experimentación

\begin{tabular}{|l|l|l|}
\hline Tratamiento & \multicolumn{1}{|c|}{$\begin{array}{c}\text { Costo Alimentación } \\
\text { Promedio / animal (COP) }\end{array}$} & $\begin{array}{c}\text { Costo Alimentación } \\
\text { Promedio / 4 animales (COP) }\end{array}$ \\
\hline T1 & 0 & 0 \\
\hline T2 & 14166,6 & 56666,3 \\
\hline T3 & 23247,8 & 92991,1 \\
\hline T4 & 21339,5 & 85358,2 \\
\hline
\end{tabular}

*No se calcularon costos debido a que no consumieron una dieta suplementaria 
González et al. [36] reportaron que el uso de plantas medicinales en la alimentación de becerras influyo en el incremento del consumo de alimento, mejora en la ganancia de peso y reducción en los costos de producción especialmente en los animales donde se administró extracto de cítricos.

\section{CONCLUSIONES}

La elaboración de un alimento mediante el proceso de fermentación en estado sólido permite mejorar el valor nutritivo, evidenciándose un aumento significativo en el porcentaje de proteína y otros nutrientes cuando hay un medio favorable, lo que posibilita la inclusión de subproductos orgánicos con el fin de disminuir los costos y aumentar el valor nutricional de estos para su uso en la alimentación animal. Por otra parte, la incidencia de enfermedades es menor cuando a los animales se les suministra un suplemento tipo FES, debido a que estos alimentos presentan un mayor número de microorganismos.

En este orden de ideas, el T2 que fue suplementado con $6 \mathrm{~g} / \mathrm{kg} / \mathrm{PV}$ del alimento FES papa:zanahoria, obtuvo los mejores resultados en relación con el consumo de alimento, ganancia de peso, peso final y alzada a la cruz; en términos económicos, este tratamiento fue más económico al representar un ahorro de \$9081,2 COP en relación con la suplementación con concentrado comercial, convirtiéndolo en una alternativa de alimentación para terneros reduciendo los costos de producción y aumentando la eficiencia de los animales. Sin embargo, el uso de dosis más altas del alimento FES, no mostro mejor resultado, siendo su rendimiento inferior al del concentrado comercial por lo cual se hace necesario realizar más estudios para determinar las cantidades óptimas a suministrar del alimento y diferentes subproductos agrícolas y pecuarios.

\section{REFERENCIAS}

[1] CLOVIS-OLIVEIRA, KLEVERTON; FACHINELLO, ARLEI-LUIZ. Crescimento da demanda mundial de alimentos e restrições do fator terra no Brasil. Revista de Economia e Sociologia Rural, v. 56, n. 2, 2018, p. 195-212. https://doi.org/10.1590/1234-56781806-94790560201

[2] CASTILLO-BADILLA, GLORIANA; VARGAS-LEITÓN, BERNARDO; HUECKMANN-VOSS, FRANK; ROMERO-ZÚÑIGA, JUAN-JOSÉ. Factores que afectan la producción en primera lactancia de vacas lecheras de Costa Rica. Agronomía Mesoamericana, v. 30, n. 1, 2019, p. 209-227. http://dx.doi.org/10.15517/am.v30i1.33430

[3] LÓPEZ-VARELA, DAVID. Caracterización bromatológica de pellets elaborados a partir de subproductos agropecuarios para la alimentación de bovinos. Revista Tecnología en Marcha, v. 30, 2017, p. 73-81. https://doi.org/10.18845/tm.v30i5.3226

[4] CHEBAIBI, SALIMA; LERICHE-GRANDCHAMP, MATILDE; BURGÉ, GRÉGOIRI; CLÉMENT, TIPHAINE; ALLAIS, FLORENT; LAZIRI, FATIHA. Improvement of protein content and decrease of anti-nutritional factors in olive cake by solid-state fermentation: A way to valorize this industrial by-product in animal feed. Journal of bioscience and bioengineering, v. 128, n. 3, 2019, p. 384-390.

https://doi.org/10.1016/j.jbiosc.2019.03.010

[5] FONSECA-LÓPEZ, DANIA; SAAVEDRA-MONTAÑÉZ, GABRIEL; RODRÍGUEZ-MOLANO, CARLOS-EDUARDO. Elaboración de un alimento para ganado bovino a base de zanahoria (Daucus carota L.) mediante fermentación en estado sólido como una alternativa ecoeficiente. Revista Colombiana de Ciencias Hortícolas, v. 12, n. 1, 2018, p. 175-182.

https://doi.org/10.17584/rcch.2018v12i1.7416

[6] CAICEDO, W.; FERREIRA, F.N.; VIÁFARA, DERWIN; GUAMAN, ANDREA; SOCOLA, CAROLINA; MOYANO, J.C. Composición química y digestibilidad fecal en cerdos del fruto de chontaduro (Bactris gasipaes Kunth) fermentado. Livestock Research for Rural Development, v. 31, n. 9, 2019, p. 140. 
[7] BORRAS, LUIS-MIGUEL; RODRÍGUEZ, CARLOS-EDUARDO; RODRÍGUEZ, ÁNGELA. Evaluation of calcium carbonate $\left(\mathrm{CaCO}_{3}\right)$ inclusion in solid-state kinetic fermentation of Solanum tuberosum postharvest waste. Revista Colombiana de Ciencias Hortícolas, v. 11,n. 1, 2017, p. 143-150. http://dx.doi.org/10.17584/rcch.2017v11i1.6145

[8] SAAVEDRA-MONTAÑEZ, GABRIEL-FERNANDO; CALA-GUERRERO, DEISY-CAROLINA; RODRÍGUEZ-MOLANO, CARLOS-EDUARDO. Evaluación de subproductos agrícolas sometidos a fermentación en estado sólido. Bistua Revista De La Facultad De Ciencias Básicas, v. 16, n. 1, 2019, p. 13-27.

[9] RODRÍGUEZ-SALGADO, ÁNGELA-MIREYA; BORRÁS-SANDOVAL, LUIS-MIGUEL; RODRÍGUEZ-MOLANO, CARLOS-EDUARDO. Compositional quality of food obtained with solid state fermentation: potato and carrot. Revista Colombiana de Ciencias Hortícolas, v. 13, n. 1, 2019, p. 8531-8531. https://doi.org/10.17584/rcch.2019v13i1.8531

[10] INCHAUSTI, DANIEL; TAGLE, EZEQUIEL, C. Bovinotecnia: Exterior y razas. Buenos Aires (Argentina): El Ateneo, 1982.

[11] AGUIRRE, L.A.; RODRÍGUEZ, ZORAYA; BOUCOURT, R.; SACA, V.; SALAZAR, R.; JIMÉNEZ, M. Efecto del suero de leche en la fermentación en estado sólido de la pulpa de café (Coffea arabica L.) para uso en la alimentación de rumiantes. Cuban Journal of Agricultural Science, v. 52, n. 3 , 2018, p. 303-312.

[12] BREA-MAURE, ODELIN; ELÍAS-IGLESIAS, ARABEL; ORTIZ-MILÁN, ABEL; MOTTA-FERREIRA, WALTER; HECHAVARRÍA-RIVIAUX, SUSET. Efecto de la urea y del tiempo en la fermentación en estado sólido de la harina de frutos del árbol del pan (Artocarpus altilis). Ciencia y Agricultura, v. 12, n. 2 , 2015, p. 91-101. https://doi.org/10.19053/01228420.4395

[13] CAICEDO, WILLAM; MOYA, CARLOS; TAPUY, ANDREA; CAICEDO, MARIA; PEREZ, MANUEL. Composición química y digestibilidad aparente de tubérculos de taro procesados por fermentación en estado sólido (FES) en cerdos de crecimiento. Revista de Investigaciones Veterinarias del Perú, v. 30, n. 2, 2019, p. 580-589. https://doi.org/10.15381/rivep.v30i2.16078

[14] FONSECA-LÓPEZ, DANIA; MONROY-FONSECA, LILY; RODRÍGUEZ-MOLANO, CARLOS-EDUARDO. Efecto del método de extracción del almidón de Canna indica L. sobre sus propiedades físico-químicas. Revista Lasallista de Investigación, v. 16, n. 2, 2019, p. 44-52. https://doi.org/10.22507/rli.v16n2a4

[15] YOHE, T.T.; SCHRAMM, H.; PARSONS, C.L.M.; TUCKER, H.L.M.; ENGER, B.D.; HARDY, N.R.; DANIELS, K.M. Form of calf diet and the rumen. I: Impact on growth and development. Journal of Dairy Science, $v$. 102, 2019, p. 8486-8501. https://doi.org/10.3168/jds.2019-16449

[16] PAZOKI, A.; GHORBANI, G.R.; KARGAR, S.; SADEGHI-SEFIDMAZGI, A.; DRACKLEY, J.K.; GHAFFARI, M.H. Growth performance, nutrient digestibility, ruminal fermentation, and rumen development of calves during transition from liquid to solid feed: Effects of physical form of starter feed and forage provision. Animal Feed Science and Technology, v. 234, 2017, p. 173-185. https://doi.org/10.1016/j.anifeedsci.2017.06.004

[17] ENGELKING, L.E.; MATSUBA, T.; INOUCHI, K.; SUGINO, T.; OBA, M. Effects of feeding hay and calf starter as a mixture or as separate components to Holstein calves on intake, growth, and blood metabolite and hormone concentrations. Journal of Dairy Science, v. 103, n. 5, 2020, p. 4423-4434. https://doi.org/10.3168/jds.2019-17676

[18] BEIRANVAND, H.; GHORBANI, G.R.; KHORVASH, M.; NABIPOUR, A.; DEHGHAN-BANADAKY, M.; HOMAYOUNI, A.; KARGAR, S. Interactions of alfalfa hay and sodium propionate on dairy calf performance and rumen development. Journal of Dairy Science, v. 97, n. 4, 2014, p. 2270-2280. https://doi.org/10.3168/jds.2012-6332

[19] CASTRO-FLORES, PAMELA; ELIZONDO-SALAZAR, JORGE-ALBERTO. Crecimiento y desarrollo ruminal en terneros alimentados con iniciador sometido a diferentes procesos. Agronomía Mesoamericana, v. 23, n. 2, 2012, p. 343-352. 
[20] KARGAR, S.; KANANI, M. Reconstituted versus dry alfalfa hay in starter feed diets of Holstein dairy calves: Effects on feed intake, feeding and chewing behavior, feed preference, and health criteria. Journal of Dairy Science, v. 102, n. 5, 2019, p. 4061-4071. https://doi.org/10.3168/jds.2018-15189

[21] IMANI, M.; MIRZAEI, M.; BAGHBANZADEH-NOBARI, B.; GHAFFARI, M.H. Effects of forage provision to dairy calves on growth performance and rumen fermentation: A meta-analysis and meta-regression. Journal of dairy science, v. 100, n. 2, 2017, p. 1136-1150.

[22] MIRZAEI, M.; KHORVASH, M.; GHORBANI, G.R.; KAZEMI-BONCHENARI, M.; GHAFFARI, M.H. Growth performance, feeding behavior, and selected blood metabolites of Holstein dairy calves fed restricted amounts of milk: No interactions between sources of finely ground grain and forage provision. Journal of dairy science, v. 100, n. 2, 2017, p. 1086-1094.

[23] OMIDI-MIRZAEI, H.; AZARFAR, A.; KIANI, A.; MIRZAEI, M.; GHAFFARI, M.H. Interaction between the physical forms of starter and forage source on growth performance and blood metabolites of Holstein dairy calves. Journal of dairy science, v. 101, n. 7, 2018, p. 6074-6084. https://doi.org/10.3168/jds.2017-13892

[24] KHAN, M.A.; LEE, H.J.; LEE, W.S.; KIM, H.S.; KI, K.S.; HUR, T.Y.; CHOI, Y.J. Structural growth, rumen development, and metabolic and immune responses of Holstein male calves fed milk through step-down and conventional methods. Journal of Dairy Science, v. 90, n. 7, 2007, p. 3376-3387. https://doi.org/10.3168/jds.2007-0104

[25] BEIRANVAND, H.; KHANI, M.; OMIDIAN, S.; ARIANA, M.; REZVANI, R.; GHAFFARI, M.H. Does adding water to dry calf starter improve performance during summer?. Journal of dairy science, v. 99, n. 3, 2016, p.1903-1911. https://doi.org/10.3168/jds.2015-10004

[26] SUAREZ-MENA, F.X.; HEINRICHS, A.J.; JONES, C.M.; HILL, T.M.; QUIGLEY, J.D. Digestive development in neonatal dairy calves with either whole or ground oats in the calf starter. Journal of dairy science, v. 98, n. 5, 2015, p. 3417-3431. https://doi.org/10.3168/jds.2014-9193

[27] HARRIS, T.L.; LIANG, Y.; SHARON, K.P.; SELLERS, M.D.; YOON, I.; SCOTT, M.F.; BALLOU, M. A. Influence of Saccharomyces cerevisiae fermentation products, SmartCare in milk replacer and Original XPC in calf starter, on the performance and health of preweaned Holstein calves challenged with Salmonella enterica serotype Typhimurium. Journal of dairy science, v. 100, n. 9, 2017, p.7154-7164. https://doi.org/10.3168/jds.2016-12509

[28] REZAZADEH, FATEMEH; KOWSAR, RASOUL; RAFIEE, HASSAN; RIASI, AHMAD. Fermentation of soybean meal improves growth performance and immune response of abruptly weaned Holstein calves during cold weather. Animal Feed Science and Technology, v. 254, 2019, p. 114206. https://doi.org/10.1016/j.anifeedsci.2019.114206

[29] MOJAHEDI, S.; KHORVASH, M.; GHORBANI, G.R.; GHASEMI, E.; MIRZAEI, M.; HASHEMZADEH-CIGARI, F. Performance, nutritional behavior, and metabolic responses of calves supplemented with forage depend on starch fermentability. Journal of dairy science, v. 101, n. 8, 2018, p. 7061-7072. https://doi.org/10.3168/jds.2017-13798

[30] SILVA, A.L.; MARCONDES, M.I.; DETMANN, E.; MACHADO, F.S.; VALADARES-FILHO, S.C.; TRECE, A.S.; DIJKSTRA, J. Effects of raw milk and starter feed on intake and body composition of Holstein $\times$ Gyr male calves up to 64 days of age. Journal of dairy science, v. 98, n. 4, 2015, p. 2641-2649. https://doi.org/10.3168/jds.2014-8833

[31] JIANG, X.; MA, G.M.; CUI, Z.Q.; LI, Y.; ZHANG, Y.G. Effects of fermented corn gluten meal on growth performance, plasma metabolites, rumen fermentation and bacterial community of Holstein calves during the pre-weaning period. Livestock Science, v. 231, 2020, p. 103866.

https://doi.org/10.1016/j.livsci.2019.103866

[32] KIM, M.H.; YUN, C.H.; LEE, C.H.; HA, J.K. The effects of fermented soybean meal on immunophysiological and stress-related parameters in Holstein calves after weaning. Journal of dairy science, v. 95, n. 9, 2012, p. 5203-5212.

https://doi.org/10.3168/jds.2012-5317 
[33] HERNÁNDEZ-GARCÍA, JUAN-EMILIO; SEBASTIÁN-FRIZZO, LAUREANO; RODRÍGUEZ-FERNÁNDEZ, JUAN-CARLOS; VALDEZ-PANECA, GREGORY; VIRGINIA-ZBRUN, MARIA; CALERO-HERRERA, IBRAHIN. Evaluación in vitro del potencial probiótico de Lactobacillus acidophilus SS80 y Streptococcus thermophilus SS77. Revista de Salud Animal, v. 41, n. 1, 2019, p. e09 ref.52.

[34] ALUGONGO, G.M.; XIAO, J.X.; CHUNG, Y.H.; DONG, S.Z.; LI, S.L.; YOON, I.; CAO, Z.J. Effects of Saccharomyces cerevisiae fermentation products on dairy calves: Performance and health. Journal of dairy science, v. 100, n. 2, 2017, p. 1189-1199. https://doi.org/10.3168/jds.2016-11399

[35] KHAN, M.A.; CANSADO, D.M.; VON KEYSERLINGK, M.A.G. Hay intake improves performance and rumen development of calves fed higher quantities of milk. Journal of dairy science, v. 94, n. 7, 2011, p.3547-3553. https://doi.org/10.3168/jds.2010-3871

[36] AVALOS-GONZÁLEZ, RAMIRO; PEÑA-REVUELTA, BLANCA-PATRICIA; RODRÍGUEZ-DIMAS, NORMA; ÁVILA-CISNEROS, RAFAEL; GONZÁLEZ-ÁVALOS, JOSÉ. Costos de alimentación en becerras Holstein suministrando leche entera adicionada con extracto de plantas medicinales. Revista Mexicana de Agronegocios, v. 45, 2019, p. 339-354. 